\title{
Comment on "Physical performance is associated with visual acuity in university students: results of a school-based study"
}

\author{
Yandi Li (1), Xiaofei Li* (1)
}

Dear Editor,

I was very glad to read the study by Wu et al. ${ }^{1}$ in which they demonstrated that physical exercise might help improve visual acuity and university students should practice strength exercises to improve physical performance. However, some concerns should be raised in my opinion.

To begin with, it is believed that there may be an accompanying relationship between physical performance and vision level, but not necessarily a causal relationship. The promotion of eye exercises may help prevent vision loss. Therefore, the authors may have exaggerated the relationship between physical exercise and vision. Whether physical exercise can promote vision improvement needs to be further tested by queue studies.

In addition, the author also did not account for the research object and research background. The specific location of the subjects from China, what is special here. All subjects were not described in detail, such as age, gender, family background, major, interests, and hobbies. Only by describing the general demographic characteristics in detail can we draw reliable conclusions.

\section{AUTHORS "CONTRIBUTIONS}

All authors have contributed equal to work.

\section{REFERENCE}

1. Wu KL, Yang L, Sun X, He L, Du T. Physical performance is associated with visual acuity in university students: results of a school-based study. Rev Assoc Med Bras (1992). 2020;66(3):296-9. https://doi.org/10.1590/1806-9282.66.3.296

'Yiwu Central Hospital, Department of infectious diseases - Zhejing, China.

*Corresponding author: xiaofeili2000@163.com

Conflicts of interest: the authors declare there is no conflicts of interest. Funding: none.

Received on February 05, 2021. Accepted on March 14, 2021. 